This is an author produced version of a paper published in Clinical Physiology and Functional Imaging. This paper has been peer-reviewed but does not include the final publisher proof-corrections or journal pagination.

Citation for the published paper:

Tragardh E, Arheden H, Pettersson J, Wagner GS, Pahlm O.

"Determination of the ability of high-frequency ECG to estimate

left ventricular mass in humans, determined by

magnetic resonance imaging"

Clinical Physiology and Functional Imaging, 2006, Vol: 26, Issue: 3, pp. 157-62

http://dx.doi.org/10.1111/j.1475-097X.2006.00662.x

Access to the published version may require journal subscription.

Published with permission from: Blackwell Synergy 


\title{
DETERMINATION OF THE ABILITY OF HIGH-FREQUENCY ECG TO ESTIMATE LEFT VENTRICULAR MASS IN HUMANS, DETERMINED BY MAGNETIC RESONANCE IMAGING
}

Elin Trägårdh, MD, ${ }^{\mathrm{a}}$ Håkan Arheden, $\mathrm{MD}, \mathrm{PhD},{ }^{\mathrm{a}}$ Jonas Pettersson, $\mathrm{MD}, \mathrm{PhD},{ }^{\mathrm{a}}$ Galen $\mathrm{S}$ Wagner, ${ }^{b} \mathrm{MD}$, Olle Pahlm, MD, $\mathrm{PhD}^{\mathrm{a}}$

From the a Department of Clinical Physiology, Lund University, Lund, Sweden, and ${ }^{\mathrm{b}}$ Duke University Medical Center, Durham, NC.

Short title: High-frequency ECG vs LVM in humans

Address: Elin Trägårdh

Department of Clinical Physiology

University Hospital,

SE-221 85 Lund, Sweden

Telephone: +4646173301

Fax: +4646151769

E-mail: Elin.Tragardh@med.lu.se

\begin{abstract}
Background Previous studies have shown a significantly higher correlation between left ventricular mass index (LVMi) and high-frequency QRS components (HFQRS) than between LVMi and QRS amplitudes in the standard frequency range in rabbits. The purpose of the present study was to compare electrocardiographic measurements from standard and high frequency ranges with left ventricular mass (LVM) and LVMi determined by magnetic resonance imaging in humans.

Methods 62 normal subjects were studied. Signal-averaged electrocardiograms (ECGs) from the 12 standard leads were analyzed in the standard frequency range (0.05$150 \mathrm{~Hz})$, in the middle $(25-100 \mathrm{~Hz})$ and high end $(50-150 \mathrm{~Hz})$ of the standard frequency range and in the $150-250 \mathrm{~Hz}$ range. Root mean square (RMS) values from the HF-QRS and QRS amplitude measurements from the standard ECGs were compared with LVM and LVMi.

Results The correlations between LVMi and HF-QRS were similar to those between LVMi and standard ECG. When regarding LVM, however, the correlations found in standard ECG were higher than those found in HF-QRS.

Conclusions Contrary to previous results in animals, we found in humans no better correlation between HF-QRS and LVM/LVMi than between standard ECG and LVM/LVMi.
\end{abstract}

Keywords: signal-averaged ECG, high-frequency QRS components, healthy subjects 


\section{INTRODUCTION}

The electrocardiographic diagnosis of left ventricular hypertrophy is an important clinical problem. The standard 12-lead electrocardiogram (ECG) remains one of the most widely used diagnostic tools for detecting left ventricular hypertrophy. However, based on echocardiographic standard, the sensitivity is poor at high levels of specificity, limiting the utility of the ECG for left ventricular hypertrophy detection (Devereux et al., 1984; Reichek \& Devereux, 1981).

The standard ECG by convention contains frequencies between 0.05 and $150 \mathrm{~Hz}$, but the ECG signal also includes higher frequencies (Golden et al., 1973). The highest amplitudes of the high-frequency components are found within the QRS complex (Abboud, 1993). Okin et al found a significantly higher correlation between the left ventricular mass index (LVMi) and high-frequency QRS components (HF-QRS) than between LVMi and standard ECG QRS amplitudes (Okin et al., 1992). Signal-averaged orthogonal lead recordings in normal rabbits and in those with left ventricular hypertrophy were used in their study. The high-pass filtered vector integral was significantly correlated with LVMi even among the normal rabbits. The results suggest that assessment of HF-QRS could improve the electrocardiographic diagnosis of left ventricular hypertrophy.

Magnetic resonance imaging is a three-dimensional imaging technique that is not dependent on geometric assumptions and not limited in the position or orientation of the image sections. Magnetic resonance imaging has become gold standard in the assessment of left ventricular volumes, function and mass. Excellent results for both accuracy (Caputo et al., 1987;
Maddahi et al., 1987; Lund et al., 2000) and reproducibility (Firmin et al., 1987; Semelka et al., 1990; Grothues et al., 2002) of measurements have been demonstrated.

A previous study by Carlsson et al (Carlsson et al, in press) showed a statistically significant correlation, but poor correlation coefficients, between left ventricular mass (LVM) and established left ventricular hypertrophy criteria. With the results of that study and the study by Okin et al (Okin et al., 1992) in mind, we therefore wanted to test the hypothesis that RMS values from HF-QRS correlate better with magnetic resonance imagingdetermined LVM and LVMi than does standard ECG.

\section{METHODS}

\section{Study population}

The study population consisted of 62 healthy volunteers in different age groups recruited by advertisement in the University Hospital of Lund, Sweden. Volunteers were not considered for the study if they had either clinical or ECG evidence of an old myocardial infarction. To reduce any potential confounding factors in the ECG analysis, patients with the following abnormalities on standard 12-lead ECG were not considered: intraventricular conduction delay with QRS duration $\geq$ $120 \mathrm{~ms}$, ventricular pre-excitation, left anterior fascicular block, or atrial fibrillation. Further, the individuals were normotensive (blood pressure: systolic $<140 \mathrm{~mm} \mathrm{Hg}$ and diastolic < $90 \mathrm{~mm} \mathrm{Hg}$ ) and had no direct or indirect signs of systemic or metabolic disease. The study was approved by the local committee on human research and complies with the Declaration of Helsinki. Informed consent was 
obtained from each patient prior to enrolment.

\section{Determination of left ventricular mass}

Throughout the study, magnetic resonance imaging was performed on a $1.5 \mathrm{~T}$ system (Magnetom Vision; Siemens, Erlangen, Germany) with a 25 $\mathrm{mT} / \mathrm{m}$ gradient with a phased-array body coil. All subjects were placed in supine position and imaged using a protocol described earlier (Engblom et al., 2004). In short, scout imaging was performed to enable identification of the cardiac long and short axes. Short axis cine images covering the entire left ventricle were then acquired using a turbo fast low-angle shot sequence (slice thickness $10 \mathrm{~mm}$, field of view $380 \mathrm{~mm}$, matrix $126 \times 256$, repetition time $100 \mathrm{~ms}$ (echo sharing resulting in phases every $50 \mathrm{~ms}$ ), echo delay time $4.8 \mathrm{~ms}$, flip angle $20^{\circ}$ ). The fast lowangle shot sequence was triggered by ECG, and image acquisition was undertaken during breath hold. Nine to twelve slices were required to completely cover the left ventricle, depending on heart size. Image acquisition time for the left ventricle was approximately ten minutes per subject.

The image analysis was performed with publicly available software (Scion Image Beta 4.0.2). The first frame of the cine image loop was selected as the end-diastolic image. The end-systolic image was chosen as the image containing the smallest left ventricular blood pool diameter. Endo- and epicardial borders were outlined manually on each end-diastolic and endsystolic image. The papillary muscles were included in the LVM (Pennell, 2002; Friberg et al, 2004). To obtain the LVM, the planimetered area of each slice was multiplied by slice thickness.
LVM was determined both in enddiastole and end-systole for internal validation.

Besides LVM, LVMi (LVM (g) divided by body weight (kg)) (Okin, 1992), was used in the study.

\section{ECG acquisition}

The ECGs were recorded using equipment provided by Siemens-Elema AB, Solna, Sweden. The precordial leads were obtained using the standard electrode placement while the limb leads were obtained using the MasonLikar electrode placement to reduce noise due to skeletal muscle activity (Mason \& Likar, 1966). The signals were digitized at a sampling rate of 1 $\mathrm{kHz}$, with an amplitude resolution of 0.6 $\mu \mathrm{V}$ and stored on a PC hard disc for analysis.

The ECG was acquired continuously for five minutes, at rest in supine position, within one hour after the magnetic resonance imaging.

\section{Signal averaging}

The five-minute recording from each patient was signal-averaged in order to minimize the noise level, which is essential for later analysis of the lowamplitude HF-QRS. The recordings were processed off-line, using software for ECG analysis developed by the Signal Processing Group, Department of Applied Electronics, Lund University, Sweden. For signal averaging only beats with similar morphology were accepted. Each beat in each recording was cross-correlated to a "template beat", representing the predominant morphology. In the beat alignment the highest cross-correlation value was determined by shifting each beat in relation to the template (Pahlm \& Sörnmo, 1987). Beats with crosscorrelation coefficient $<0.97$ were not included. 


\section{Analysis of different frequency ranges}

The amplitudes in the standard frequency range were automatically determined in the obtained signalaveraged ECG. The measurements used to estimate LVM were the Cornell voltage measurement $(\mathrm{R}(\mathrm{aVL})+\mathrm{S}(\mathrm{V} 3))$ (Molloy et al., 1992) the Cornell product measurement ((R(aVL) + $\mathrm{S}(\mathrm{V} 3)) *$ QRS duration) (Molloy et al., 1992) and the Sokolow-Lyon measurement (S(V1) + R(V5/6)) (Sokolow \& Lyon, 1949). Two of the individuals had a QS complex in V1, its amplitude was used for the SokolowLyon measurement.

The obtained 12-lead signalaveraged ECG was analyzed using a Butterworth filter (Proakis \& Manolakis, 1996) within the frequency intervals of $25-100 \mathrm{~Hz}, 50-150 \mathrm{~Hz}$, and $150-250 \mathrm{~Hz}$. The signal was first filtered forward and then backward to ensure linear phase of the filtering process (Proakis \& Manolakis, 1996). The QRS components in each of the 12 leads were expressed as RMS values during the entire QRS duration. RMS values were calculated by 1) squaring the amplitude of each sample, 2) determining the mean of these squares, and 3) determining the square root of this mean. The QRS onset and offset were automatically determined from the signal-averaged ECG in the standard frequency range before the filtering process (Jonson et al., 1984; Xue et al., 1995).

Trend samples of the signalaveraged ECG were obtained every 5 seconds. The noise level was calculated in each lead and expressed as an RMS value in an interval of $100 \mathrm{~ms}$, starting $100 \mathrm{~ms}$ after the QRS offset. Trend samples for further analysis were obtained after one minute of registration, if the noise level did not exceed $3.2 \mu \mathrm{V}$ in the frequency band 25-100 Hz. If the noise level exceeded $3.2 \mu \mathrm{V}$, the first subsequent trend sample with a noise level $<3.2 \mu \mathrm{V}$ was used. The cut noise levels for the frequency band $50-150 \mathrm{~Hz}$ was $1.3 \mu \mathrm{V}$ and $0.4 \mu \mathrm{V}$ for $150-250 \mathrm{~Hz}$.

For correlations with LVM, the RMS value in lead V1 and the highest RMS value in lead V5 or V6 was summed (RMS-Sokolow-Lyon). Likewise, the RMS value in lead aVL and V3 was summed (RMS-Cornell voltage), and RMS-Cornell product was calculated as QRS-duration, measured in the standard frequency range, times RMS-Cornell voltage. The correlations between LVM/LVMi and RMS-values in each individual lead were also investigated, as well as the sum of the RMS-values over all 12 leads (“Summed RMS").

\section{Statistical methods}

Continuous data were subjected to the Kolmogorov-Smirnov test to determine their distribution. Because of lack of Gaussian distribution among the RMS values, Spearman rank correlation coefficient, $\rho$, was used for correlations between LVM/LVMi and ECG measurements. A criterion level of $p<$ 0.05 was considered statistically significant. All analyses were carried out using SPSS for Windows 11.5 (SPSS Inc., Chicago, Illinois, USA).

\section{RESULTS \\ Study population}

The study population consisted of 62 individuals (28 females and 34 males). Mean age was 46.4 years (SD 15.4 years, range 21-79 years). Body mass index ranged from 15.4 to 29.7 $\mathrm{kg} / \mathrm{m} 2$ (mean $23.8 \mathrm{~kg} / \mathrm{m} 2$, SD 3.5 $\mathrm{kg} / \mathrm{m} 2$ ). The LVM varied between 96 
and $223 \mathrm{~g}$ among the individuals (mean $167 \mathrm{~g})$.

\section{Comparison between LVM and standard ECG QRS amplitudes}

The correlation between LVM and Cornell voltage was $\rho=0.31(\mathrm{p}=0.018)$, versus $\rho=0.40(p=0.002)$ between LVM and Cornell product and $\rho=0.35$ $(p=0.006)$ between LVM and SokolowLyon. The highest correlation between peak-to-peak amplitude was found in lead V3 $(\rho=0.41, p=0.001)$. Figure 1A shows the correlation between the peakto-peak amplitude in V3 and LVM.

\section{Comparison between LVM and RMS-values}

In the $25-100 \mathrm{~Hz}$ frequency range, there was a significant correlation between LVM and RMS-Cornell product $(\rho=0.35, p=0.007)$, but not between LVM and any of the other parameters (RMS values in individual leads, RMS-Cornell voltage and RMSSokolow-Lyon). Even when the two outliers in this frequency interval were excluded, the correlation was statistically significant $(\rho=0.34, p=$ $0.010)$. In the $50-150 \mathrm{~Hz}$ range, none of the correlations between RMS values and LVM was found to be significant. The highest correlation with LVM was found in lead V2 $(\rho=0.20, p=0.109)$. In the $150-250 \mathrm{~Hz}$ frequency range, the only significant correlation was between LVM and Summed RMS $(\rho=0.33, p=$ 0.011). Figure 1B shows the best correlation in this set of correlations: the correlation between RMS-Cornell product and LVM in the $25-100 \mathrm{~Hz}$ range.

\section{Comparison between LVMi and standard ECG QRS amplitudes}

The correlation between LVMi and Cornell voltage was $\rho=0.06(p=0.638)$, versus $\rho=0.08(p=0.528)$ between
LVMi and Cornell product and $\rho=0.30$ $(p=0.018)$ between LVMi and SokolowLyon. The highest correlation between LVMi and peak-to-peak amplitude was found in lead aVL $(\rho=0.36, p=0.004)$. Figure 2A shows the correlation between the peak-to-peak amplitude in aVL and LVMi.

\section{Comparison between LVMi and RMS-values}

The best correlation between LVMi and any RMS-value in the 25$100 \mathrm{~Hz}$ range was found in lead V2 $(\rho=$ $0.33, p=0.009)$. In this frequency range, the correlations in lead $\mathrm{V} 1$ and $\mathrm{aVL}$, as well as Summed RMS were also found to be statistically significant. In the 50-150 $\mathrm{Hz}$ range, the best correlation was found in lead V1 $(\rho=0.37, p=0.003)$. Here, also the correlations in lead V2, V3, Summed RMS, RMS-Sokolow-Lyon, RMS-Cornell voltage, and RMS-Cornell product were statistically significant. In the $150-250 \mathrm{~Hz}$ range, the only statistically significant correlation was found between LVMi and RMS-Cornell product $(\rho=0.26, p=0.039)$. Figure $2 B$ shows the best correlation in this set of correlations: the correlation between RMS-V1 and LVMi in the 50-150 Hz range.

\section{DISCUSSION}

It has previously been demonstrated that the high-frequency ECG correlates better with LVMi in rabbits than does the standard ECG, both when considering normal rabbits and those with left ventricular hypertrophy $(\mathrm{r}=0.71, \mathrm{p}<0.001$ in normal rabbits) (Okin et al., 1992). The present study, however, shows similar results for standard ECG and HF-QRS. Surprisingly, the established electrocardiographic criteria for detecting left ventricular hypertrophy 
were found to have a low correlation with LVMi, with only Sokolow-Lyon being statistically significant. When considering LVM, standard ECG correlated far better with LVM than did HF-QRS. The highest correlations found in the present study were between V3 and LVM $(\rho=0.41)$ and between Cornell product and LVM $(\rho=0.40)$. The results indicate that the standard frequency range is better suited to predict LVM than are higher frequency ranges. The reason for the different findings is not obvious. Okin et al used a $44 \mathrm{~Hz}$ high-pass filter and they used time-voltage integrals of the vector QRS complex instead of RMS values.

The most frequently used frequency band when studying HF-QRS has been 150-250 Hz. The only statistically significant correlation found in the present study in this frequency band was between LVM and Summed RMS. It is not clear if this is a "true" significant correlation or a type I error because of the many correlations investigated in. However, it is possible that LVM is a confounding factor for Summed RMS, which has been used in previous studies (Pettersson et al., 2000; Ringborn et al., 2001; Trägårdh et al., 2004).

A previous study (Carlsson et al., in press) showed that there is a statistically significant correlation between LVM and established electrocardiographic left ventricular hypertrophy criteria in the same healthy study population used in this study, but that QRS duration alone is more strongly correlated to LVM than are other ECG criteria.

The basic physiology of HF-QRS is not fully understood, but Abboud et al have suggested that the morphological changes in HF-QRS seen in ischemic hearts can be attributed to a slowing of conduction velocity in the region of ischemia (Abboud et al., 1991). If this is true, there is no obvious reason why patients with left ventricular hypertrophy should have higher RMS values than normal individuals.

For unknown reasons, a large inter-individual variation in RMS values has been found in the $150-250 \mathrm{~Hz}$ range (Pettersson et al., 2000). It is possible that this variation could hide any potentially real additional effects of LVM in this frequency range. However, HF-QRS have been demonstrated to be quite reproducible within individuals (Batdorf et al., 2004), which probably makes the method more useful for monitoring situations or when a previous HF-QRS recording is available.

It has been suggested that HFQRS can be a diagnostic tool in various heart diseases. It has previously been shown that diminution of HF-QRS is a more sensitive detector of acute myocardial ischemia than changes in the ST segment of standard ECG (Abboud et al., 1987; Pettersson et al., 1998; Pettersson et al., 2000). Several investigators have documented reduced HF-QRS in patients with old myocardial infarction compared to healthy individuals (Goldberger et al., 1980; Bhargava et al., 1981). Ringborn et al, however, found no difference in HF-QRS when comparing patients with ischemic heart disease, with and without old infarction (Ringbornet al., 2001). It has also been shown that this entire group of patients with ischemic heart disease has lower HF-QRS than healthy individuals (Trägårdh et al., 2004), suggesting that some pathophysiological process, other than healed myocardial infarction, diminishes HF-QRS in this group. The National Aeronautics and Space Administration has developed a software program that is able to analyze 
and display changes in HF-QRS in real time, and their results on detecting coronary artery disease are promising (Schlegel et al., 2004).

\section{Limitations of the study}

The present study only includes normal individuals. It is possible that the range of LVM is too small in the study and that a better correlation could be found if including patients with left ventricular hypertrophy as well.

There are previously defined criteria for determining left ventricular hypertrophy in the standard ECG. In other frequency bands, however, no such criteria have been established. In the present study, RMS analogs of established standard ECG criteria for detecting left ventricular hypertrophy were used for estimating LVM in the $25-100, \quad 50-150$ and $150-250 \mathrm{~Hz}$ intervals, as were also RMS values from individual leads. It is possible that other measurements in these frequency bands correlate better with LVM.

\section{In summary}

Contrary to previous results in animals, we found no better correlation between HF-QRS and LVM/LVMi than between standard ECG and LVM/LVMi in healthy human individuals.

\section{ACKNOWLEDGEMENTS}

This study was supported by the Swedish Heart Lung Foundation. 


\section{REFERENCES}

1. Abboud S, Cohen RJ, Selwyn A, Ganz P, Sadeh D, Friedman PL. Detection of transient myocardial ischemia by computer analysis of standard and signal-averaged high-frequency electrocardiograms in patients undergoing percutaneous transluminal coronary angioplasty. Circulation (1987); 76: 585-596.

2. Abboud S, Berenfeld O, Sadeh D. Simulation of high-resolution QRS complex using a ventricular model with a fractal conduction system. Effects of ischemia on high-frequency QRS potentials. Circ Res (1991); 68: 1751-1760.

3. Abboud S. High-frequency electrocardiogram analysis of the entire QRS in the diagnosis and assessment of coronary artery disease. Prog Cardiovasc Dis (1993); 35: 311-328.

4. Batdorf NJ, Feiveson AH, Schlegel TT. Month-to-month and year-to-year reproducibility of high frequency QRS ECG signals. J Electrocardiol (2004); 37: 289-296

5. Bhargava V, Goldberger A. Myocardial infarction diminishes both low and high frequency QRS potentials: power spectrum analysis of lead II. J Electrocardiol (1981); 14: 57-60.

6. Caputo GR, Tscholakoff D, Sechtem U, Higgins CB. Measurement of canine left ventricular mass by using MR imaging. AJR Am J Roentgenol (1987); 148: 33-38.

7. Carlsson M, Trägårdh E, Engblom H, Wagner G, Pahlm O, Arheden H. Left ventricular mass by 12-lead ECG in healthy subjects: Comparison to cardiac magnetic resonance imaging. $J$ Electrocardiol (2006); 39: 67-72.

8. Devereux RB, Casale PN, Eisenberg RR, Miller DH, Kligfield P. Electrocardiographic detection of left ventricular hypertrophy using echocardiographic determination of left ventricular mass as the reference standard. Comparison of standard criteria, computer diagnosis and physician interpretation. J Am Coll Cardiol (1984); 3: 82-87.

9. Engblom H, Hedström E, Palmer J, Wagner GS, Arheden H. Determination of the left ventricular long-axis orientation from a single short-axis MR image: relation to BMI and age. Clin Physiol Funct Imaging (2004); 24: 310-315.

10. Firmin DN, Nayler GL, Klipstein RH, Underwood SR, Rees RS, Longmore DB. In vivo validation of magnetic resonance velocity imaging. J Comput Assist Tomogr (1987); 11: 751-756.

11. Friberg P, Allansdotter-Johnsson A, Ambring A, Ahl R, Arheden H, Framme J, Johansson A, Holmgren D, Wahlander H, Marild S. Increased left ventricular mass in obese adolescents. Eur Heart J (2004); 25: 987-992.

12. Goldberger AL, Bhargava V, Froelicher V, Covell J, Mortara D. Effect of myocardial infarction on the peak amplitude of high frequency QRS potentials. J Electrocardiol (1980); 13: 367-372.

13. Golden DP Jr, Wolthuis RA, Hoffler GW. A spectral analysis of the normal resting electrocardiogram. IEEE Trans Biomed Eng (1973); 20: 366-372.

14. Grothues F, Smith GC, Moon JC, Bellenger NG, Collins P, Klein HU, Pennell DJ. Comparison of interstudy reproducibility of cardiovascular magnetic resonance with two-dimensional echocardiography in normal subjects and in patients with heart failure or left ventricular hypertrophy. Am J Cardiol (2002); 90: 29-34.

15. Jonson B, Lundh B, Pahlm O, Tranesjö J. Determination of QRS onset and end in orthogonal and scalar ECGs. A new approach. IEEE Computer Society, Computers in Cardiology (1984); 459462.

16. Lund GK, Wendland MF, Shimakawa A, Arheden H, Stahlberg F, Higgins CB, Saeed M. Coronary sinus flow measurement by means of velocity-encoded cine MR imaging: Validation by using flow probes in dogs. Radiology (2000); 217: 487-493.

17. Maddahi J, Crues J, Berman DS, Mericle J, Becerra A, Garcia EV, Henderson R, Bradley W. Noninvasive quantification of left ventricular myocardial mass by gated proton nuclear magnetic resonance imaging. J Am Coll Cardiol (1987); 10: 682-692.

18. Mason RE, Likar I. A new system of multiple-lead exercise electrocardiography. Am Heart $J$ (1966); 71: 196-205.

19. Molloy TJ, Okin PM, Devereux RB, Kligfield P. Electrocardiographic detection of left ventricular hypertrophy by the simple QRS voltage-duration product. J Am Coll Cardiol (1992); 20: 11801186.

20. Okin PM, Donnelly TM, Parker TS, Wallerson DC, Magid NM, Kligfield P. High-frequency analysis of the signal-averaged ECG. Correlation with left ventricular mass in rabbits. $J$ Electrocardiol (1992); 25: 111-118.

21. Pahlm O, Sörnmo L. Data processing of exercise ECGs. IEEE Trans Biomed Eng (1987); 34: 158165. 
22. Pennell DJ. Ventricular volume and mass by CMR. J Cardiovasc Magn Reson (2002); 4: 507-513.

23. Pettersson J, Lander P, Pahlm O, Sörnmo L, Warren SG, Wagner GS. Electrocardiographic changes during prolonged coronary artery occlusion in man: comparison of standard and highfrequency recordings. Clin Physiol (1998); 18: 179-186.

24. Pettersson J, Carro E, Edenbrandt L, Maynard C, Pahlm O, Ringborn M, Sörnmo L, Warren SG, Wagner GS. Spatial, individual, and temporal variation of the high-frequency QRS amplitudes in the 12 standard electrocardiographic leads. Am Heart J (2000); 139: 352-358.

25. Pettersson J, Pahlm O, Carro E, Edenbrandt L, Ringborn M, Sörnmo L, Warren SG, Wagner GS. Changes in high-frequency QRS components are more sensitive than ST-segment deviation for detecting acute coronary artery occlusion. J Am Coll Cardiol (2000); 36: 1827-1834.

26. Proakis JG, Manolakis DG. Digital Signal Processing - Principles, Algorithms, and Applications (1996). Upper Saddle River, NJ: Prentice-Hall.

27. Reichek N, Devereux RB. Left ventricular hypertrophy: relationship of anatomic, echocardiographic and electrocardiographic findings. Circulation (1981); 63: 1391-1398.

28. Ringborn M, Pahlm O, Wagner GS, Warren SG, Pettersson J. The absence of high-frequency QRS changes in the presence of standard ECG QRS changes of old myocardial infarction. Am Heart $J$ (2001); 14: 573-579.

29. Schlegel TT, Kulecz WB, DePalma JL, Feiverson AH, Wilson JS, Rahman MA, Bungo MW. Real-time 12-lead high-frequency QRS electrocardiography for enhanced detection of myocardial ischemia and coronary artery disease. Mayo Clin Proc (2004); 79: 339-350.

30. Semelka RC, Tomei E, Wagner S, Mayo J, Kondo C, Suzuki J, Caputo GR, Higgins CB. Normal left ventricular dimensions and function: interstudy reproducibility of measurements with cine MR imaging. Radiology (1990); 174: 763-768.

31. Sokolow M, Lyon TP. Ventricular complex in left ventricular hypertrophy as obtained by unipolar precordial and limb lead. Am Heart J (1949); 37: 161-186.

32. Trägårdh E, Pahlm O, Wagner GS, Pettersson J. Reduced high-frequency QRS components in patients with ischemic heart disease compared to normal subjects. J Electrocardiol (2004); 37: 157-162.

33. Xue Q, Reddy S, Aversano T. Analysis of high-frequency signal-averaged ECG measurements. $J$ Electrocardiol (1995); 28 (Suppl): 239-245. 


\section{FIGURES}

Figure 1. Scatter plots of the best correlation between measurements based on standard ECG and LVM (A) and between HF-QRS and LVM (B). The best correlation between standard ECG and LVM was found to be the peak-to-peak amplitude in lead V3 and between HF-QRS and LVM RMS-Cornell product in the $25-100 \mathrm{~Hz}$ range.

A

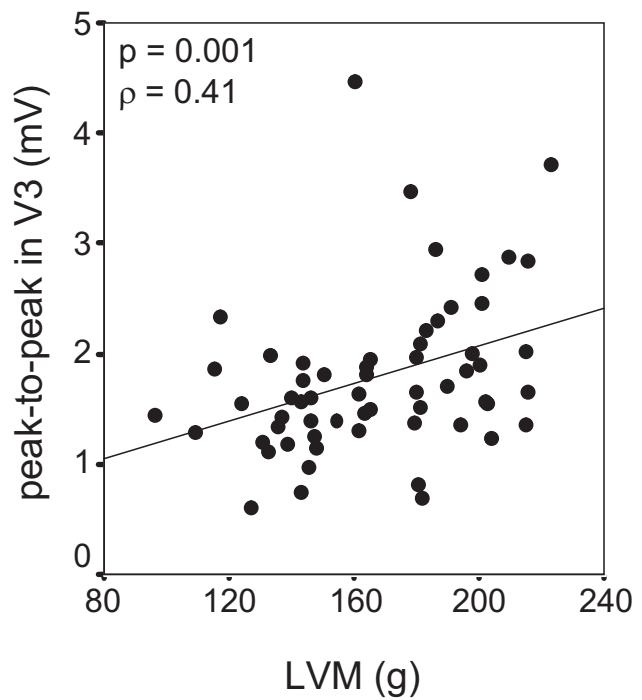

B

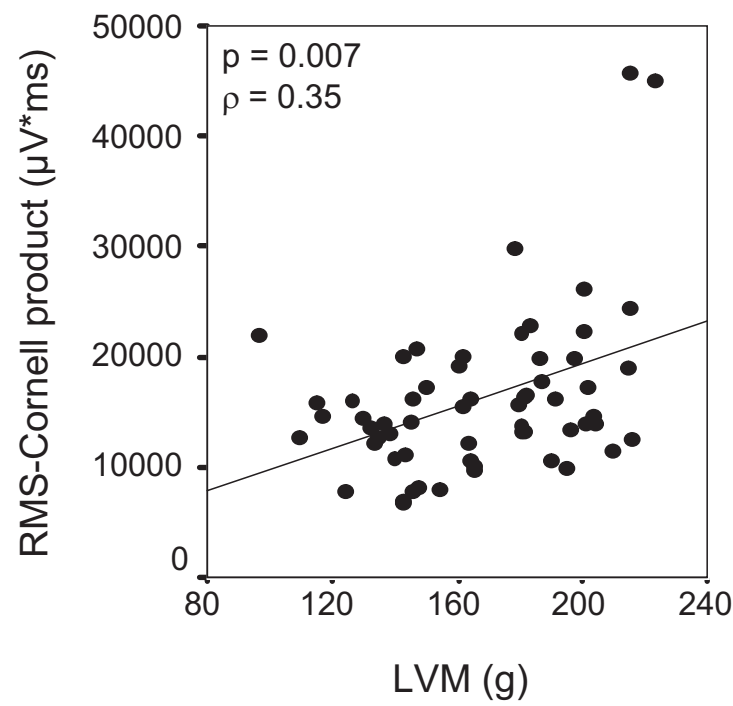

Figure 2. Scatter plots of the best correlation between measurements based on standard ECG and LVMi (A) and between HF-QRS and LVMi (B). The best correlation between standard ECG and LVMi was found to be the peak-to-peak amplitude in lead aVL, and between HF-QRS and LVMi RMS-V1 in the 50-150 Hz range.

A

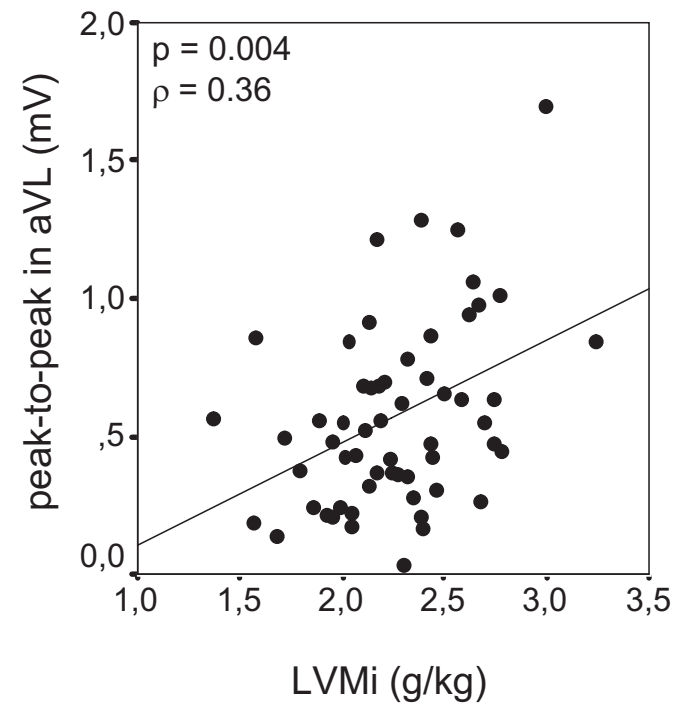

B

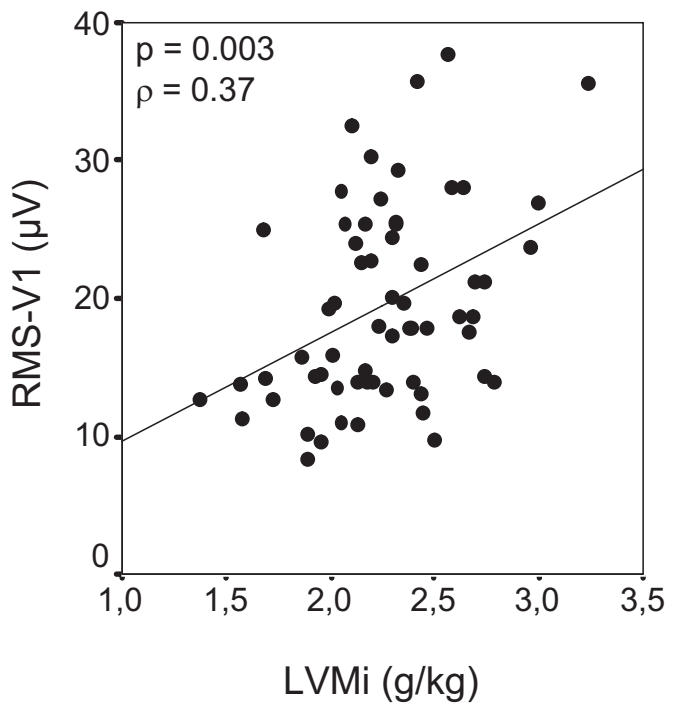

